\title{
Engineering in Architecture Schools: Some Principles and Cases
}

\author{
Emanuel Jannasch \\ Dalhousie University School of Architecture \\ jannasch@dal.ca
}

\begin{abstract}
The training and professional responsibilities of architects and engineers are widely divergent. But it falls to the architect as prime consultant to try and bridge this gap. This paper describes some of the methods used by architecture schools to teach engineering content in a manner that fosters effective collaboration.
\end{abstract}

\section{Introduction}

Engineering and architecture are separated by a deep culture gap. Whereas architects can be characterized as wishy-washy generalists, engineering is rigorous and specialized. Education in the two fields reinforces this pattern. Architecture schools are required to impart some appreciation of engineering design, but the reverse is almost unheard of. And whereas the architect is inclined to see such exclusion as narrowminded and even arrogant, for engineers it is simply a mark of professional respect for professional boundaries. From an engineer's perspective, the attempts made to teach engineering within architecture are largely qualitative and at best superficial. But architecture schools face a sharp paradox. No matter how well their engineering courses are taught, an architecture student can never learn what their engineering colleagues acquire through full time study. And yet, in professional practice they are expected to act as prime consultant, providing direction and leadership to a project team. How can this condition be resolved? How can an architect learn to fulfill this obligation to their client and to their engineering sub-consultants? This paper will present some basic principles and some case studies, not because engineering design has anything to learn from other design disciplines, but simply because in those branches of engineering concerned with building design, some communication with architects will need to take place.

\section{The Problem With Architecture}

When designing a dam the engineer can be confident that the water will configure itself to the structure, but an architect has a more complicated problem. In a building, the configuration of the contained medium takes precedence over - yet is limited by - the design of the containing structure. More complicated still, the fabric of the building comprises several material systems must serve the contained spaces effectively, in spite of conflicting spatial demands. Furthermore, they should be collectively configured so as to improve the volumetric characteristics of each space at the same time as they subtract volume from the building. In non-architectural construction the use of the building is either sufficiently utilitarian or sufficiently generalized that volumetric or formal refinement are not part of the equation. For architects, integrating systems for technical performance and cost is only a starting point. Their principal responsibility is to the volumetric quality of the habitable spaces and the formal qualities of the building fabric. Engineers are seldom asked to concern themselves with anything beyond the most simplistic aspects of spatial quality or formal expression, although it is worth noting that this statement applies better to North America than to Latin America or Europe. So how can one train architects to manage their sub-consultants in such a way that will enable them to contribute to a building of architectural merit?

\subsection{Three Approaches}

Engineering educators in architecture tend to one of three directions. Traditionally, every effort was made to raise the bar if not to a professional level, at least as high as possible. Instructors were typically drawn from the engineering profession, and students learned to derive methods of analysis from abstract principles in mathematics and in strength of materials. At best this approach generated redundant skills, at worst students would transfer classroom compliance with their instructor to uncomprehending deference to their 
professional sub-consultants. Nowadays, architectural education is more likely to realize that an architects understanding of technical questions needs to be different than an engineer's. At its most simplistic, this approach emphasizes "alternative" structures drawn from anywhere but standard engineering construction. Anything from product design to animal physiology, from the great structures of the nineteenth century to musical instruments, from aerospace to algorithmic graphics may provide visual inspiration for technical form. Of course, all these realms are valuable sources of inspiration for engineers as well as architects, but they must be understood as physical systems not as mere imagery. A third approach to architectural technology begins with the reality of architectural practice. It helps students respect the strict conceptual boundaries within which engineering practice is undertaken, and helps them place that practice into the richer context of values and diverse modes of thought which architecture must address.

\section{Single Systems}

The great challenge of architecture is to integrate building systems with respect to incommensurable technical and aesthetic criteria. However, students can't be expected to address a problem of this complexity without some understanding of the individual systems concerned

\subsection{Field Trip}

Highway 102 passes over, under, and near a great variety of bridges illustrating the wide variety of forms that can afford a simple function. Large beams are seen to accommodate varying bending moment by varying web depth, flange depth, or flange thickness. They observe first hand how physical context affects the engineering solution. They are also asked to consider invisible factors: how the relative cost of steel versus concrete and of materials versus construction time might contribute to design decisions, and what kinds of equipment would have been needed to fabricate, transport, and erect the structural components. Maintainability and inspectability are also discovered to be vital design considerations. In the design of bearings we can see changing attitudes to mechanical necessity and mechanical articulation, and here and there we even observe decisions made purely for aesthetic reasons. [Insert Fig. 1A] [Insert Fig. 1B] These principles could have been taught from a theoretical basis and illustrated by the instructor's slides, but the time and expense of a field trip are justified by at least three considerations.
A) Reinforcing the principle that theories, diagrams, images, and numerical models are approximations of reality, not the other way round. There is always more information to be gleaned from reality than there is from abstraction, and that the responsibility of design professional is to reality. B) We can see the effects of design and construction problems that may not be visible in last year's slides. C) An architect needs to understand the perception as well as the physical performance of things; the human impact of size, scale, and form can only be judged in reality.

\subsection{Schematic Modelling}

Architects often work on structures small enough that they fall easily under prescriptive code requirements, but complex enough to obscure their structural behaviour. Whether they will seek an engineers assistance or not for the final sizing, architects do need to understand structural configuration. Load path models in which students identify only those members required to transfer one specific point load to the ground are an excellent analytical tool. Ideally they are assigned as a pair, treating vertical loads and horizontal loads respectively. Horizontal models are displayed pinned to the wall, so that loading may be more easily visualized.

\subsection{Responsible Rules of Thumb}

Engineering instruction scrupulously avoids numerical approximations which apply in one context or at one magnitude scale and yet break down in others. But in their role of systems initiators, architects must often work with preliminary approximations. In the 70's, Edward Allen at MIT began to develop powerful and responsibly contextualized rules of thumb. $\mathrm{He}$ presented them in graphical forms that allowed many variables to be perused simultaneously. Originally circulated as photocopies, eventually these were redrawn and compiled as the Architects Studio Companion. The example shown combines span, corrugation depth, slab depth and loading. It allows the architect to identify a range of possibilities within which the engineer can do more rigorous optimization. [Insert Fig. 2]

\subsection{Transparent Math}

Students often evaluate proposed buildings by testing numerical approximations of their designs against mathematical models of physical behavior, generally structural or thermal. Often the mathematical models are built by others, and in the case of commercial 
packages the structure of the models can be quite invisible. Such exercises are counterproductive. Employing models they don't understand, students never appreciate the number and the impact of assumptions in building and using numerical models, and how such assumptions can promote particular design traits.

Rather than training students in the naïve use of notionally sophisticated models, it may be wiser to train them in the intelligent application of simple models. In one exercise, students are asked to compare a cubical three story house with an elongated one story configuration of the same floor area and ceiling height. The one story version has significantly more surface area, with apparent savings of energy. Then, step by step, students factor in the lower $\partial \mathrm{T}$ of the slab, the relative ease of insulating the attic, the difficulty of insulating and draft-proofing floor/wall intersections, and account for stack effect and wind shear as modeled for different site conditions. The point is not to find a correct evaluation of the building, but to develop some sense of the relative importance of design variables, and how this scale of factors varies with context. More importantly they see how different ways of modeling a building can encourage different kinds of building form, and that it is not possible to select a reasonable approach to modelling before one has a reasonably complete sense of the design - and even then one needs to choose a modelling system with a critical awareness of its biases. Architects' professional responsibility is not necessarily to know how to run a given evaluation package, but to be able to evaluate the evaluation. [Insert Fig. 3]

\subsection{War Stories}

The fundamental principle that a system is not a piece of reality but an abstraction bears plenty of repetition. The following example is less concerned with the inclusion of secondary numerical factors than with the basic configuration of a system. It also takes the form of a $\mathrm{n}$ anecdote from the instructor's professional experience. These can illustrate the complexities of practice better than any number of theories or built works.

In this instance the architect wanted to add a large skylight at mid-span of an aged and lightly framed flat roof. The proposal was to remove the decking in way of the skylight but to leave the joists running through; the intent being to avoid reframing, to exhibit part of the original structure, and to diffuse and distribute daylight. The project engineer was unwilling to allow the load of the gabled skylight to be placed on the marginal existing joists, as it weighed considerably more than the deck to be removed, and would tend to increase any snow loads. The architect on the other hand felt that doubling the through joists would destroy the architectural value of the proposed intervention. He pointed out that since the ridge of the skylight was at right angles to the joists, the load on the through joists was a) taken away from mid span and landed closer to the joist bearing, and b) because the skylight incorporated a ridge beam, half the load was carried to the gable end, which could be borne on built up members without affecting the visible joists. The engineer agreed that the skylight need not be considered a monolithic load, and the through joists were left in the elegant form originally proposed.. The value of such stories, whether taken from mechanical as well as structural or other cases, is that while an architect is seldom qualified to question an engineer's numerical work, they may well be in a position to assess how well a numerical model matches to reality, and they should have the confidence to contribute their thoughts on the subject. [Insert Fig. 4]

\section{Coordinating Multiple Systems}

Designing a novel system of any complexity is a difficult task at best, and virtually impossible within the constraints of an architect's fee. Yet this is often what an architect is asked to do. Having a large repertoire of established systems configurations becomes essential, either to draw upon directly, or to help establish general principles that may be judiciously applied to new patterns of integration.

\subsection{BSI Handbook}

The AIA Building Systems Integration Handbook addresses the problem of arranging structural, mechanical, envelope, and interior systems in workable three dimensional configurations. The systems theory proposed as an analytical and synthetic tool suffers from profound logical flaws, nonetheless the book contains a unique catalogue of patterns of integration, together with important notes concerning advantages and disadvantages in various contexts. Familiarity with the catalogue allows students to look at other drawings or buildings with a similarly analytic eye.

\subsection{Schematic Case Studies}

Students may be asked to build a schematic model of a noteworthy building, emphasizing not a single system but the three dimensional nesting of the principal physical components of the building. Tracing each system from source to sink allows students to draw 
general lessons about the configuration of systems, while tracing their relative paths: sometimes parallel, sometimes divergent, sometimes intersecting gives a sense of that complex physiology that is in the purview of none of the individual consulting engineers, yet which the architect must manipulate in accordance with the engineers' various judgements to simultaneously establish and service appropriately configured spaces.

\subsection{Detail Case Studies}

Structural engineers who are concerned only with the structural performance of the systems they are designing may freely delegate detailing to the fabricator, to an offshore consultant, or to anyone legally qualified to do the job. This option is not open to architects. Architectural details must typically answer to several performance requirements while at the same time articulating the aesthetic expression of their building. This is an integrated and integrating task. [Insert Fig. 5]

\section{4 large Scale Models}

When students build physical models of spatial and systems arrangements they are often asked to build them at as small a scale as possible. This helps the students focus on configuration rather than detail. Of course, to select and include pertinent detail is part of the challenge. One relative measure of success in model making has been expressed as specific thinking , measured, presumably as ideas per cubic inch.

But students are also asked to build models with a very low "specific intelligence". The examples shown are structural models in the order of 1:10 or 1:5. It is doubtful whether any purely structural lessons are learned here that could not be taught through a smaller model or even a virtual model. The real lesson is that of magnitude itself. In the real world more is different. Large volumes of a material behave differently than smaller volumes. 1:5 connections are not full scale connection, but they are not merely glue, and they illustrate the principles of scaling. Erection does not require a crane, but must be carefully planned. Wind and rain have had their effects. Respect for the complexity and above all the unexpectedness of the real world is engendered. The real world is not only inanimate. As Professor Steve Mannell recently pointed out, "Things like group work, logistics, budgets, skeptics, and so on are all aspects of real world projects." [Insert Fig. 6]

\section{Collaborative Practice}

We have distinguished architects and engineers as generalists and specialists. We can extend this distinction in observing that Engineering thinking is goal oriented, convergent thinking; and that Engineering economy is based largely on achieving explicit goals with a minimum of means. Architectural thinking on the other hand is value oriented, exploratory thinking. Architectural economy is developed by maximizing those implicit values that emerge not from a problem statement directly, but only once a new system is conceived and configured. This divergence can make effective collaboration difficult, as neither party has any real understanding of the values to which the other is committed.

\subsection{Professional Practice Modules}

Architecture schools are required by their certifying bodies to make some efforts to address this culture gap. As well as inviting engineers as sessional and guest instructors, or even, in larger schools, including them on the full time faculty, schools will include coursework and workshops specifically dedicated to knowledge of and practice in various systems of collaborative and interdisciplinary, and integrative design.

\subsection{Free labs}

Several American architectural schools have incorporated full scale design build studios into their programs. Other schools on this continent experiment with more restricted versions of design build, such as Dalhousie's freelabs As in mechanical engineering projects, the purpose is to confront full scale real world conditions. Seldom can architectural goals be reduced to a race or an obstacle course. Many freelabs incorporate the ultimate complexity: a real world client embodied not in a single being but in a community group. [Insert Fig. 7]

\subsection{Best Practice Indicators}

The job of coordinating technical sub-consultants to achieve some measure of aesthetic quality in a building can be a daunting one. Perhaps what distinguishes the two fields most sharply is that for an engineer, aesthetics are only one item on a list of performance mandates that they aren't trained or expected to consider. What distinguishes the work of the architect is not merely that items like aesthetics, 
product semantics, and the languages of fine art are on their list of concerns. It is that these concerns cannot be treated as add-ons, they inhere in the substance and in the conduct of the whole job. This divergence of focus can lead to considerable difficulties in practice, but these difficulties are the prime consultant's to solve. As well as providing them with models of and experience in integrated practice, students are familiarized with a canon of engineering works that do show some appreciation of the integral nature of architectural aesthetics. Traditionally, North American schools draw their examples from the work of European engineers like Nervi, Maillart, Menn, and Torroja, Latin Americans like Dieste, or European trained practitioners like Ammann. A notable exception from Canada is Morden Yolles who trained and practiced here, but who did pursue an integrative design vision.

Models of practice such as these do make it possible for architects to imagine a more thoroughgoing collaboration with engineers, and this can be especially important when working in smaller centers. For the foreseeable future, however, the task of establishing such a collaboration would appear to remain in the architect's hands. 